\title{
Electronic epidemiological query on admission: one year results of an e-health based tool designed to risk assessment and infection control
}

\author{
C Palos ${ }^{*}$, A Bispo \\ From 3rd International Conference on Prevention and Infection Control (ICPIC 2015) \\ Geneva, Switzerland. 16-19 June 2015
}

\section{Introduction}

Patients admitted to hospitals represent a threat as they may be infected or colonized by Epidemiologically Important Microrganisms (EIM). Their detection allows screening and isolation procedures and should start early, preferably at Emergency Room (ER).

\section{Objectives}

Assessment of the results obtained from 1 year of Electronic Epidemiological Query on Admission (EEQA) fulfillment.

\section{Methods}

EEQA is a 8 question tool obligatory fulfilled at admission. It automatically generates orders (swabs, Cdiff toxin and GDH), isolation procedures, e-mails, and ultimately allows detection of colonization/infection by EIM (MRSA, Carbapenem-resistant Acinetobacter baumanii, VRE), or infection by Clostridium difficile or pulmonary Mycobacterium tuberculosis.

\section{Results}

On 2014, 13.893 EEQA were fulfilled. 25\% resulted positive. Question (Q)1 (Previous hospital or institutional stay of $>3$ days, or tracheostomy) contributed to $87,4 \%$. Q2 (EIM present on admission) was positive on 7,5\%. Q3 (Dialysis, chemotherapy or immunossupressive therapy on the last 3 months) was detected on $10,8 \%$. Q4 (Pulmonary Tuberculosis suspected or confirmed) contributed to 1,4\%. Q5 (Confirmed pneumonia caused by Group A Strepto, Mycoplasma, Adenovirus or H. influenza. Flu, Meningitis... confirmed or suspected) was positive on 0,7\% of cases. Q6 (Diarrhea associated with antibiotics administration on the last month, or in the context of contact with other patients with diarrhea, or in patients older than $65 \mathrm{y}$ without other cause) represented 3,5\%. Q7 (Diarrhea without context defined on question 6, or any exsudative wound or drain without containment) was present on 3,1\%. Q8 (Post-transplantaplasia) contributed to $0,2 \%$ of positive EEQA.

\section{Conclusion}

EEQA is a simple tool aimed to early detection of high risk patients for infection or colonization by EIM, allowing early and adequate selective screening and isolation procedures according to international guidelines [1], even before micro results are known. This protects healthcare workers, students, other patients and visitors from exposure to these patients, thus minimizing the risk for nosocomial infections.

\section{Disclosure of interest}

None declared.

Published: 16 June 2015

Reference

1. Guideline for Isolation Precautions: Preventing Transmission of Infectious Agents in Healthcare Settings. 2007.

doi:10.1186/2047-2994-4-S1-P279

Cite this article as: Palos and Bispo: Electronic epidemiological query on admission: one year results of an e-health based tool designed to risk assessment and infection control. Antimicrobial Resistance and Infection Control 2015 4(Suppl 1):P279. 